\title{
Poliolefinas reforzadas con óxido de grafeno reducido. Mediciones viscoelásticas
}

\author{
Carlos A. Guerrero Salazar, Mayra Llamas Hernández, \\ Antonio Elizondo Martínez, M. Edgar Reyes Melo, Tania E. Guerrero Salas \\ Universidad Autónoma de Nuevo León, Facultad de Ingeniería Mecánica y Eléctrica \\ carlos.guerreros@uanl.mx, mi.llamashr@gmail.com, jose.elizondomz@gmail.com, \\ reyesmeloedgar@yahoo.fr, tegs_1234@gmail.com
}

\section{RESUMEN}

Se analiza el efecto de óxido de grafeno reducido y del óxido de polietileno sobre las propiedades viscoelásticas de HDPE y PP. Los gráficos de la viscosidad dinámica presentan curvas muy similares en todos los casos. Utilizando porcentajes de rGO de $0.1 \%$ con $2 \%$ de PEO, se obtienen valores del módulo elástico cuatro veces más grandes que los de HDPE puro y tres veces más que el del PP puro. Con $0.5 \%$ de rGO en ambos composites, manteniendo el mismo porcentaje de PEO, se obtiene un módulo elástico tres veces más grande que el de las poliolefinas puras.

\section{PALABRAS CLAVE}

Grafeno, Composites, Viscoelasticidad, Polietileno de Alta Densidad, Polipropileno.

\begin{abstract}
The effect of the reduced graphene oxide and the Poly-Ethylene Oxide on the viscoelastic properties of HDPE and PP are analyzed. The results of the dynamic viscosity are very similar in all cases. Using percentages of $r G O$ of $0.1 \%$ with $2 \%$ of $P E O$, obtained values of elastic modulus for composite are four times than that of neat HDPE and three times than neat PP modulus. With $0.5 \%$ of $r G O$ in both composites, maintaining the same percentage of PEO, the elastic module obtained is three times larger than those for two neat polyolefins.
\end{abstract}

\section{KEYWORDS}

Graphene, Composites, Viscoelasticity, High Density Polyethylene, Polypropylene.

\section{INTRODUCCIÓN}

Las poliolefinas son polímeros formados a partir de alquenos (hidrocarburos insaturados que disponen de moléculas con al menos un enlace doble carbono-carbono) tales como el etileno, propileno, buteno, isopreno, y penteno, o bien de copolímeros y modificaciones derivadas. Las poliolefinas son el grupo más numeroso de termoplásticos, siendo los más importantes el polietileno y el polipropileno, debido principalmente a sus propiedades como tenacidad, elongación, facilidad de procesamiento, bajo peso y bajo costo, entre otras. ${ }^{1-3}$ Estas propiedades hacen de ellos materiales muy versátiles, ya que se pueden extruir, moldear y soplar, pudiendo utilizarse en una gran diversidad de aplicaciones. Sin embargo, para otro tipo de aplicaciones, $v g$., eléctricas, mecánicas, térmicas, estas 
propiedades remarcables no bastan, por lo que tienen que ser mejoradas o, peor aún, se tiene que cambiar de material.

Una alternativa que se ha seguido para mejorar las propiedades de las poliolefinas consiste en mezclarlas con diferentes tipos de partículas o fibras, buscando una sinergia entre las propiedades de los diferentes materiales. Esto da origen a los materiales llamados "composites", los cuales consisten en la unión física de dos o más materiales que tienen propiedades físicas y químicas diferentes, pero al combinarlos, producen un nuevo material con características muy diferentes a las que poseen los originales. La estructura y morfología de un composite está formada principalmente por una fase conocida como matriz y al menos otra fase dispersa en aquella, la cual se conoce como refuerzo o carga. El material empleado como matriz puede ser un polímero, un metal o un cerámico y la fase dispersa puede ser orgánica o inorgánica y en forma de partículas, fibras, hojuelas, etc. ${ }^{4-6}$

En las últimas décadas, entre los materiales más usados como refuerzo se encuentra el carbono y sus alótropos, $v g$., grafito, ${ }^{7,8}$ nanotubos, ${ }^{8-10}$ grafeno y sus derivados, ${ }^{11-14}$ principalmente. Lo anterior debido a que una cantidad muy pequeña de carbono o sus alótropos puede incrementar significativamente las propiedades de la matriz polimérica, aunado a que, en este tipo de refuerzos, su peso es mucho menor comparado con aquellos cerámicos o metálicos..$^{15}$

El grafeno es un material formado por una o varias capas de átomos de carbono $(<10)$ arreglados en forma hexagonal y empacados en una red 2-D en forma de panal de abeja, Se le considera el bloque de construcción básico de los fullerenos, nanotubos y grafito. ${ }^{16}$ Figura 1. Dado su tamaño nanométrico, el grafeno posee propiedades mecánicas, térmicas y eléctricas remarcables. Sin embargo, fabricar composites de grafeno o sus derivados con poliolefinas es difícil, dada la naturaleza no polar de estas últimas, por lo que las placas de grafeno tienden a aglomerarse, exhibiendo una adhesión interfacial pobre.

Este trabajo analiza el efecto de la incorporación de concentraciones pequeñas de grafeno $(<1 \%$ peso $)$ sobre las propiedades viscoelásticas del polietileno de alta densidad (HDPE) y del polipropileno (PP). Se explora también el efecto de la adición de un agente compatibilizante, el polióxido de etileno (PEO), en las propiedades del composite.

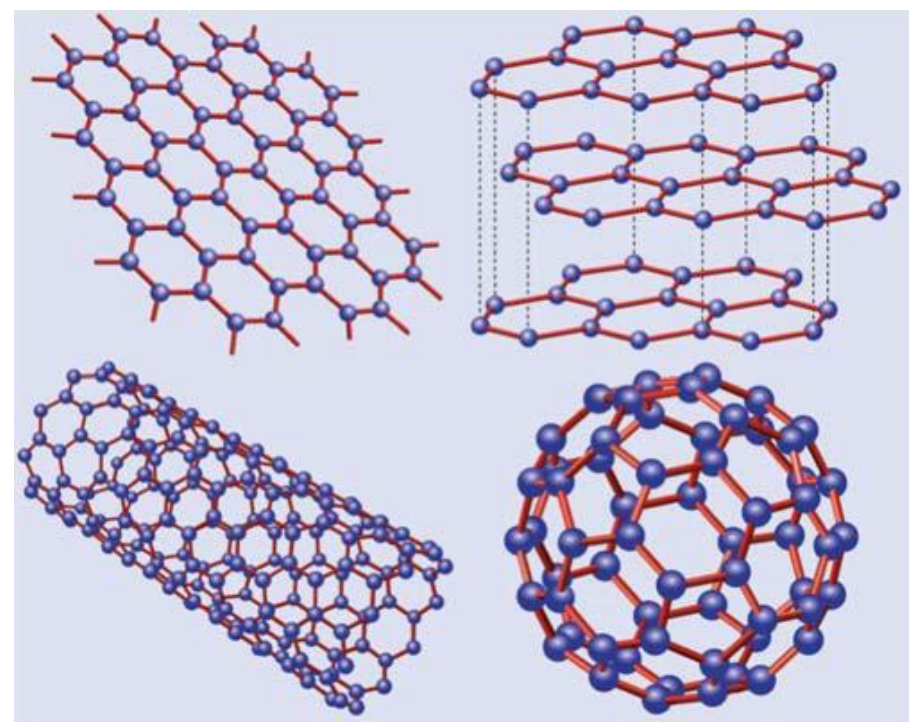

Fig. 1. El grafeno es un material 2-D que se considera como el bloque de construcción básico de otras formas grafíticas. Se puede deformar en esfera, generando los fullerenos (0-D), enrollar para formar nanotubos (1D) o apilar una capa sobre otra, formado el grafito (3-D). ${ }^{16}$ 


\section{EXPERIMENTACIÓN}

\section{Materiales utilizados}

Las poliolefinas seleccionadas para este trabajo consisten en un polietileno de alta densidad (Nova Chemicals) y un polipropileno (LyondellBasell). Las propiedades de estos materiales se enlistan en la tabla I. Para la obtención del grafeno, se usó grafito en polvo con un tamaño de partícula del orden de $20 \mu \mathrm{m}$. Este material se oxidó con permanganato de potasio $\left(\mathrm{KMnO}_{4}\right)$, nitrato de sodio $\left(\mathrm{NaNO}_{3}\right)$, ácido sulfúrico $\left(\mathrm{H}_{2} \mathrm{SO}_{4}\right)$ al $98 \%$, peróxido de hidrógeno $\left(\mathrm{H}_{2} \mathrm{O}_{2}\right)$ e hidróxido de sodio $(\mathrm{NaOH})$. Todos los químicos provienen de Sigma Aldrich, grado reactivo y se usaron como se recibieron. Después de la oxidación, el GO se redujo usando hidrosulfito de sodio $\left(\mathrm{Na}_{2} \mathrm{~S}_{2} \mathrm{O}_{4}\right)$ como agente reductor. En parte de la experimentación se utilizó como agente compatibilizante polióxido de etileno (PEO, Sigma Aldrich) del cual se reporta un peso molecular, Mw, de 600,000 g/mol.

Tabla I. Propiedades de las poliolefinas utilizadas. Datos del proveedor.

\begin{tabular}{|l|c|c|c|c|}
\hline \multicolumn{1}{|c|}{ Poliolefina } & Proveedor & $\rho\left(\mathrm{g} / \mathrm{cm}^{3}\right)$ & $\mathrm{Mw}(\mathrm{g} / \mathrm{mol})$ & $\mathrm{T}_{\mathrm{f}}\left({ }^{\circ} \mathrm{C}\right)$ \\
\hline $\begin{array}{l}\text { Polietileno de alta densidad } \\
\text { (HDPE, Sclair 19A) }\end{array}$ & Nova Chemicals & 0.96 & 126,000 & 129 \\
\hline Polipropileno (PP, Profax 6523) & LyondellBasell & 0.90 & 82,700 & 164 \\
\hline
\end{tabular}

$\rho=$ densidad; $M w$ = peso molecular; $T_{f}=$ temperatura de fusión

\section{Obtención de Óxido de Grafeno Reducido (rGO)}

La producción de las láminas de grafeno mediante la reducción química del óxido de grafito se realizó siguiendo el procedimiento previamente descrito por los autores en otras publicaciones. ${ }^{17,18}$ La ruta más común para exfoliar el grafito es mediante el uso de agentes fuertemente oxidantes, produciendo así el óxido de grafeno (GO), el cuál es un compuesto de carbono hidrofílico y no conductor. El GO se puede exfoliar fácilmente en agua usando ultrasonido. Para oxidar el grafito se siguió el método de Hummers mejorado, ${ }^{19}$ con el cual el grafito se mezcla con permanganato de potasio $\left(\mathrm{KMnO}_{4}\right)$ y una mezcla concentrada 9:1 de ácido sulfúrico y ácido fosfórico $\left(\mathrm{H}_{2} \mathrm{SO}_{4} / \mathrm{H}_{3} \mathrm{PO}_{4}\right)$. Este método proporciona un material altamente oxidado. Para recuperar las propiedades del grafeno, el GO se tiene que reducir, formándose lo que se conoce como óxido de grafeno reducido, rGO. Con la intención de evitar el uso de sustancias altamente tóxicas, reactivas y de difícil manipulación, como la hidracina y sus derivados, se utilizó como agente reductor el hidrosulfito de sodio, $\mathrm{Na}_{2} \mathrm{~S}_{2} \mathrm{O}_{4}$, Este proceso de reducción se usa en la industria textil, pero tiene el suficiente potencial para llevar a cabo la síntesis del rGO a gran escala. Para la reducción, el GO se deposita en sustratos de vidrio y se sumerge en una solución de hidrosulfito de sodio, hidróxido de sodio y agua destilada. Después de la reacción de reducción, el rGO se lava con agua destilada para eliminar residuos de la solución reductora y se seca en un horno al vacío. Una vez lavado y secado, el rGO se pulveriza en un mortero convencional, quedando el polvo listo para usarse en la preparación de los composites mediante la técnica de mezclado en fundido. La figura 2 representa el proceso de producción de las láminas de grafeno.

Para mejorar la interacción interfacial entre el rGO y la matriz poliolefínica, se realizó un segundo set experimental en donde, antes de reducir al GO, se combinó con un agente compatibilizante, siendo éste el Poli-Óxido de Etileno (PEO). Se agrega lentamente $1 \mathrm{~g}$ de PEO en polvo a $10 \mathrm{ml}$ de agua destilada a una temperatura de $80{ }^{\circ} \mathrm{C}$, mezclándose lentamente con un agitador magnético hasta obtener una pasta viscosa. Manteniendo la agitación, se disminuye la temperatura a $40^{\circ} \mathrm{C}$ para 
después agregar gota a gota $50 \mathrm{ml}$ de la solución acuosa de GO que se obtuvo después del ultrasonido. La pasta viscosa resultante se somete a un baño de ultrasonido durante 30 minutos para asegurar una buena dispersión. Después de esto, la pasta se coloca en una caja Petri para secarse en un horno de vacío a $60{ }^{\circ} \mathrm{C}$ durante 6 horas. El GO/PEO se saca de la caja Petri y se sumerge durante una hora en la solución reductora de hidrosulfito de sodio, procediendo de la misma manera a como se describió en el párrafo anterior.

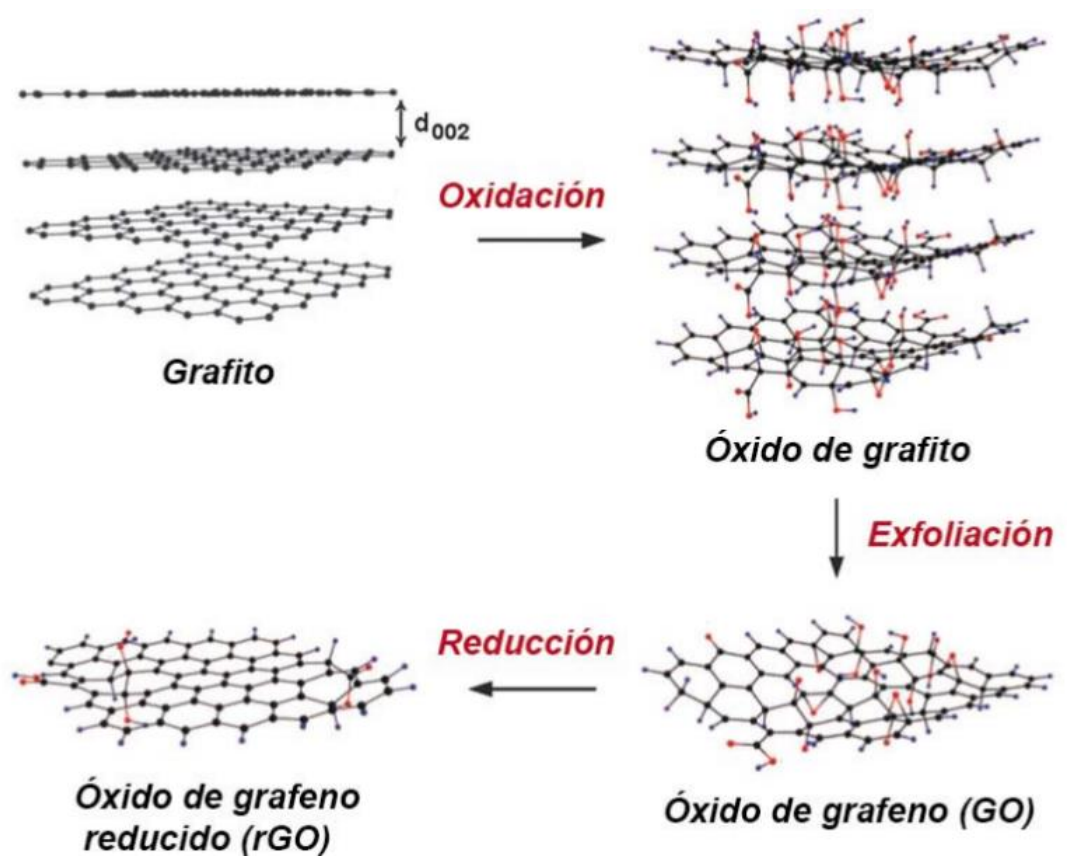

Fig. 2. Representación esquemática del proceso de producción del rGO. ${ }^{20}$

\section{Composites Poliolefinas-rGO}

Los nanocomposites poliolefínicos se fabricaron empleando la técnica de mezclado intensivo en fundido, para lo cual se usó un mezclador C.W. Brabender de doble husillo. Todo el proceso se llevó a cabo bajo una atmósfera de nitrógeno para evitar la oxidación de las muestras. La cámara del mezclador se calienta a la temperatura de trabajo, poniéndose a girar los rotores a 100 RPM. Cuando la cámara alcanza la temperatura deseada, $160^{\circ} \mathrm{C}$ para el $\mathrm{HDPE}$ o $180^{\circ} \mathrm{C}$ para el PP, se añade la poliolefina en forma gradual. Después de que se alcanza el estado estable, se alimenta el rGO, o el rGO/PEO en su caso, y se deja funcionando el sistema otros $10 \mathrm{~min}$. Al final de esta etapa, el material se retira de la cámara de mezclado y se seca. La tabla II muestra la composición de los diferentes composites formulados.

\section{Caracterización de los materiales}

El óxido de grafeno reducido se caracterizó mediante la técnica de difracción de rayos X (PANalytical $\mathrm{X}^{\prime}$ Pert Pro) con fuente de radiación $\mathrm{CuK} \alpha(\lambda=0.15418 \mathrm{~nm})$. El voltaje usado fue de $50 \mathrm{KV}$, a una velocidad de $0.5^{\circ} \mathrm{min}^{-1}$ y con un ángulo de barrido, $2 \theta$, de 1 a $40^{\circ}$. Se disolvió en etanol rGO en polvo, sometiéndose a ultrasonido; algunas gotas de esta solución se colocaron en una rejilla de cobre y se analizaron por microscopia electrónica de transmisión (JEOL, JEM-2100F) a un voltaje de aceleración de $200 \mathrm{KV}$. 
Tabla II. Composición de los composites formulados

\begin{tabular}{|c|c|c|c|c|}
\hline MUESTRA & $\begin{array}{c}\text { HDPE, } \\
\text { wt\% }\end{array}$ & $\mathrm{PP}, \mathrm{wt} \%$ & $\begin{array}{l}\text { rGO, } \\
\text { wt\% }\end{array}$ & $\begin{array}{l}\text { PEO, } \\
w t \%\end{array}$ \\
\hline PE10 & 99.9 & 0 & \multirow{2}{*}{0.1} & \multirow{2}{*}{0} \\
\hline PP10 & 0 & 99.9 & & \\
\hline PE50 & 99.5 & 0 & \multirow{2}{*}{0.5} & \multirow{2}{*}{0} \\
\hline PP50 & 0 & 99.5 & & \\
\hline PE12 & 97.9 & 0 & \multirow{2}{*}{0.1} & \multirow{2}{*}{2.0} \\
\hline PP12 & 0 & 97.9 & & \\
\hline PE15 & 94.9 & 0 & \multirow{2}{*}{0.1} & \multirow{2}{*}{5.0} \\
\hline PP15 & 0 & 94.9 & & \\
\hline PE52 & 97.5 & 0 & \multirow{2}{*}{0.5} & \multirow{2}{*}{2.0} \\
\hline PP52 & 0 & 97.5 & & \\
\hline PE55 & 94.5 & 0 & \multirow{2}{*}{0.5} & \multirow{2}{*}{5.0} \\
\hline PP55 & 0 & 94.5 & & \\
\hline
\end{tabular}

Para los composites, se evaluaron sus propiedades viscoelásticas midiendo la viscosidad compleja de las muestras en estado fundido, para lo cual se usó un reómetro rotacional de placas paralelas (Rheometric Scientific, SR-5000). Las pruebas fueron isotérmicas $\left(180^{\circ} \mathrm{C}\right)$ y de tipo oscilatorio, en un rango de frecuencias de 0.1 a $100 \mathrm{rad} / \mathrm{s}$. Todas las pruebas se realizaron bajo atmósfera de nitrógeno. Se analizaron también muestras sólidas, para lo cual se usó un analizador mecánico dinámico (Perkin Elmer DMA 8000) en modo tensión; se evaluaron los módulos y la tan $\delta$ en función de la temperatura, en un intervalo de $25^{\circ} \mathrm{C}$ a $160^{\circ} \mathrm{C}$, con una frecuencia constante de 0.5 y $1.0 \mathrm{~Hz}$.

\section{RESULTADOS Y DISCUSIÓN}

\section{Óxido de grafeno reducido}

La figura 3 muestra los difractogramas obtenidos para el grafito y para el rGO sintetizado. En el primer caso, es notorio un intenso pico en un ángulo de $26.38^{\circ}$, el cual corresponde a la reflexión en el plano (002) de la estructura grafítica. ${ }^{21}$ La distancia interplanar calculada de de $0.337 \mathrm{~nm}$. Sin embargo, para el rGO no se aprecia ningún pico en el difractograma. Esto se puede atribuir a que las láminas del rGO que se obtienen no poseen una orientación uniforme y se encuentran bastante desordenadas, lo que disminuye considerablemente las interferencias constructivas de los rayos $\mathrm{X}$ difractados, por lo que se infiere que la diferencia entre los dos difractogramas se debe a que en el rGO no existen arreglos tridimensionales de largo alcance. Estos datos están en concordancia con resultados reportados previamente en otros trabajos..$^{21,22}$

En otras mediciones que no se reportan en este trabajo, se obtuvo también el difractograma del GO, observando que la intensidad del pico en $26.46^{\circ}$ disminuye considerablemente, apareciendo un nuevo pico en $11.30^{\circ}$ lo cual se ocasiona debido a la expansión entre las capas de grafito por la introducción de grupos funcionales que contienen oxígeno ${ }^{22}$ y la consecuente distorsión del arreglo hexagonal de los átomos de carbono del grafito. Sin embargo, se sigue observando un pico menos intenso a $26.69^{\circ}$, indicando que la oxidación del grafito no fue completa. La distancia interplanar para el GO fue de $0.782 \mathrm{~nm}$, mucho mayor a la del grafito debido a, como ya se mencionó, la expansión entre las capas al anclarse los grupos funcionales que contienen oxígeno. 


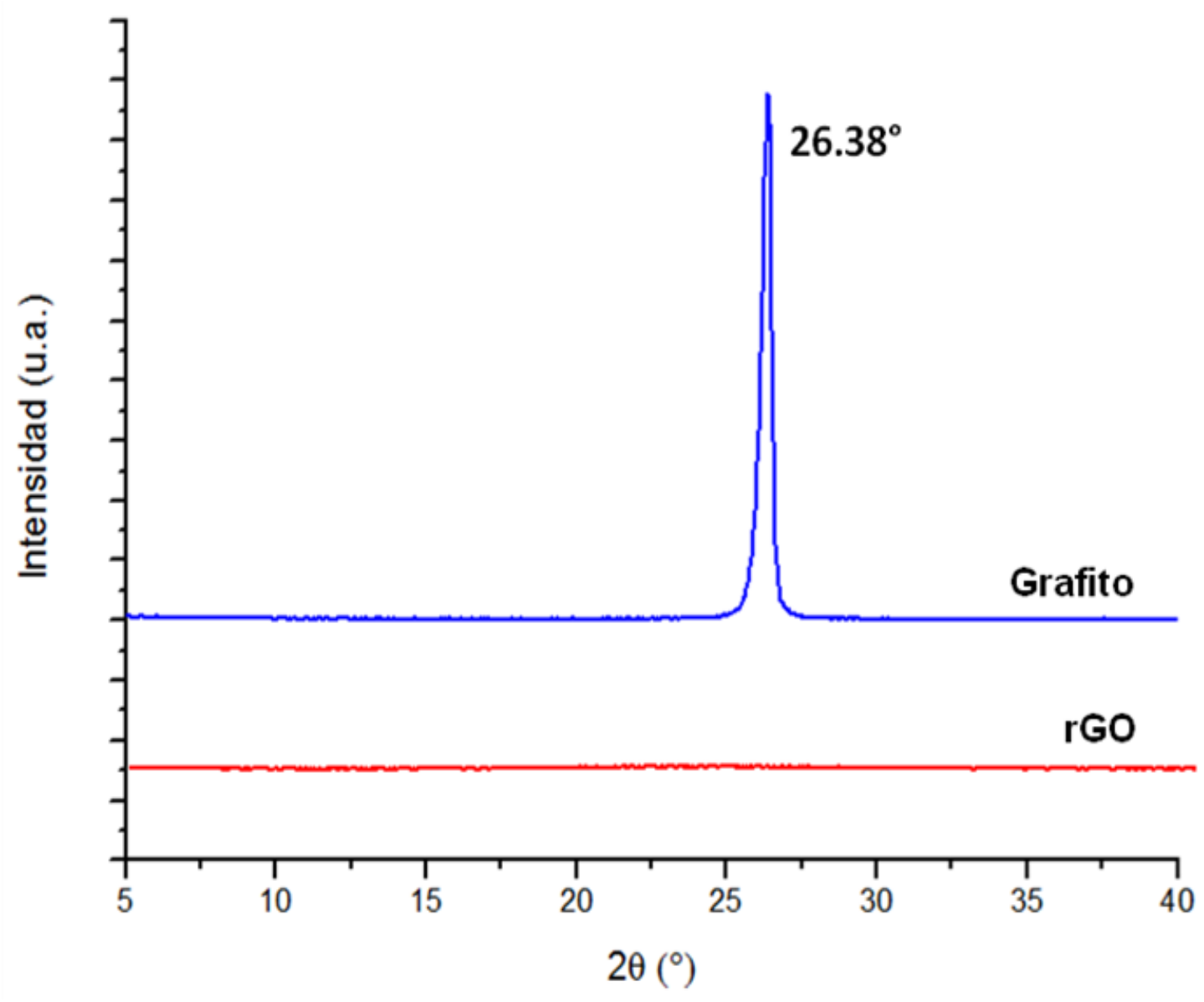

Fig. 3. Difractogramas de rayos $X$ para el grafito y el rGO sintetizado.

Las imágenes de Microscopia electrónica de transmisión (TEM) de la figura 4 corresponden a nanoestructuras de rGO. En ellas se puede observar láminas de grafeno multicapa superpuestas, las cuales coinciden con las que han sido reportadas previamente en la literatura. ${ }^{23}$ La textura de las hojas es altamente cristalina con pocos defectos, debido a las etapas de ultrasonido y centrifugado. La morfología del rGO se asemeja a nanoláminas corrugadas. En las figuras es posible apreciar zonas más oscuras, lo que muy probablemente esté asociado a un aumento en el número de láminas apiladas. Las líneas que se observan en algunas secciones podrían representar láminas de grafeno agrupadas que posiblemente, después de eliminar el solvente que las mantenía separadas, colapsaron y se mantuvieron unidas durante y después del proceso de reducción química.

\section{Composites Poliolefinas-rGO}

La viscosidad dinámica permite conocer el comportamiento visoelástico del material bajo fuerzas oscilatorias de corte durante los procesos de transformación de polímeros. La figura 5 muestra las mediciones de la viscosidad dinámica ( $\eta$ ') para las poliolefinas vírgenes y los composites PoliolefinarGO. El fin del comportamiento Newtoniano, que corresponde a relajaciones tipo líquido, se aprecia a bajas frecuencias, tanto para el PP como para el HDPE y sus composites. 

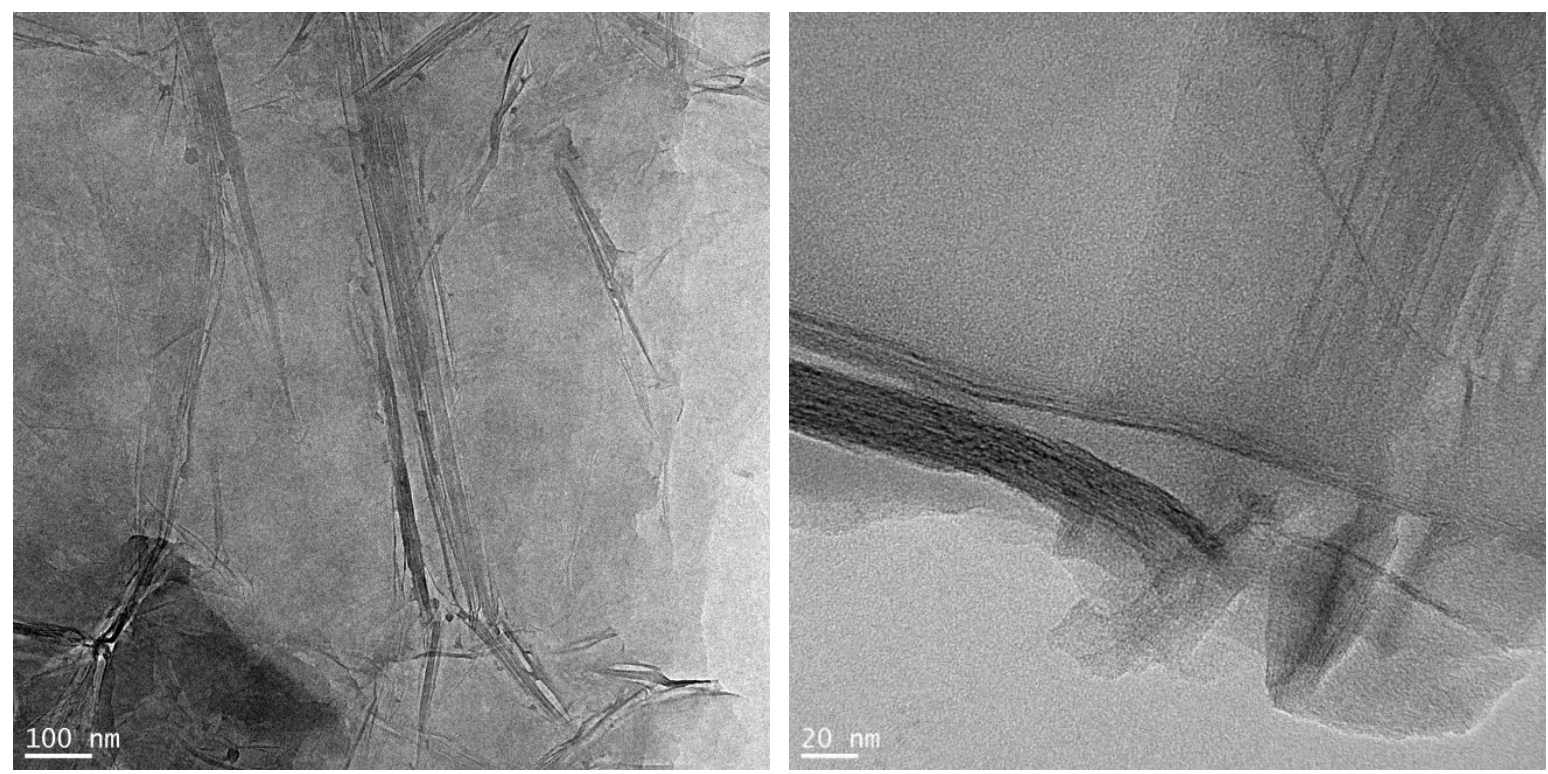

Fig. 4. Imágenes de rGO obtenidas mediante microscopia electrónica de transmisión.

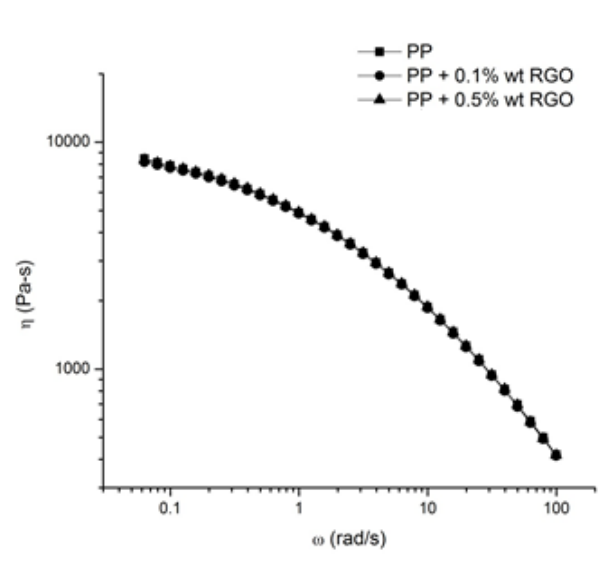

(a)

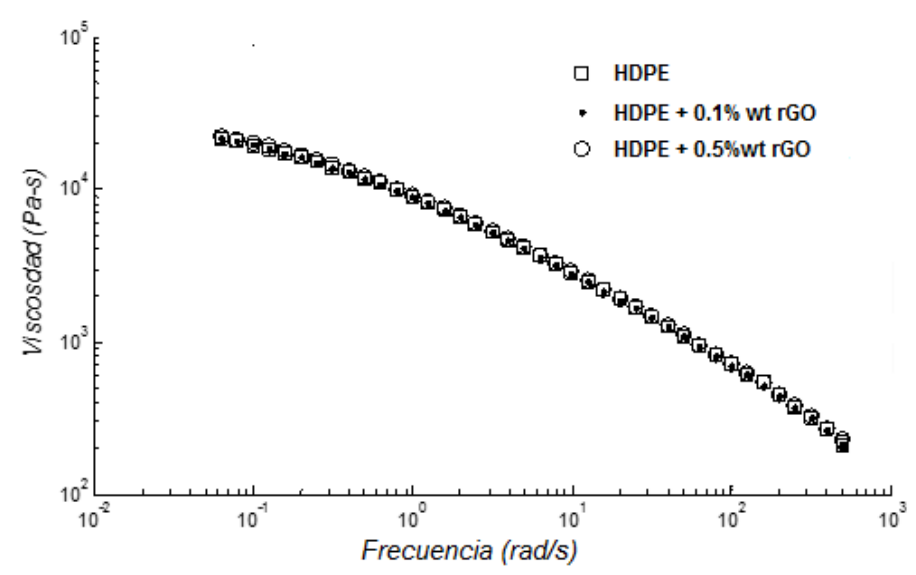

(b)

Fig. 5. Variación de la parte real de la viscosidad compleja con la frecuencia para los composites (a) Polipropileno-óxido de grafeno reducido y (b) Polietileno de alta densidad-óxido de grafeno reducido.

Para frecuencias arriba de $10^{\circ}$, y para todos los materiales analizados, se aprecia el comportamiento pseudoplástico, disminuyendo la viscosidad a medida que aumenta la frecuencia. Es evidente que la viscosidad de la matriz, HDPE y PP, no se ve grandemente influenciada por las cantidades adicionadas de $\mathrm{rGO}$, las cuales fueron muy pequeñas, implicando una falta de interacción entre el refuerzo y la matriz. La existencia de grupos funcionales en base a oxígeno que subsistieron en el rGO y a la naturaleza no polar de las dos poliolefinas pudiera ser otra causa de la aparente falta de interacción. Se esperaba que al añadir el PEO como compatibilizante, el rGO y la poliolefina tuvieran una mejor interacción interfacial, lo cual se vería reflejado en una mayor oposición al flujo y, por ende, en un incremento en la viscosidad de las muestras. Sin embargo, la figura 6 presenta un 
comportamiento muy similar al observado en las muestras sin compatibilizante, en donde la curva de viscosidad de las poliolefinas puras es prácticamente igual a la de los composites con PEO y rGO. Dado que en estos resultados no se alcanza a apreciar el efecto del refuerzo y del compatibilizante en la matriz plástica, se procedió a evaluar el móduo elástico de los composites, con y sin compatibilizante.

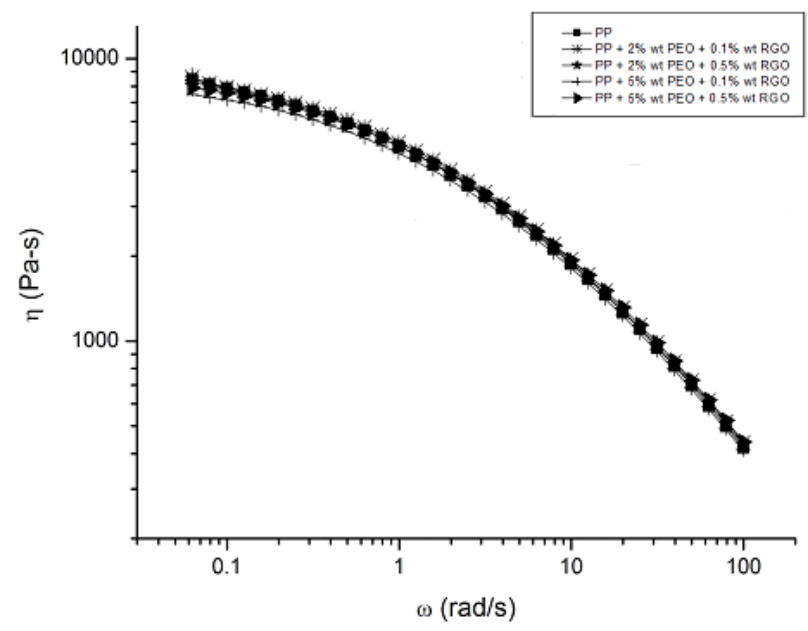

(a)

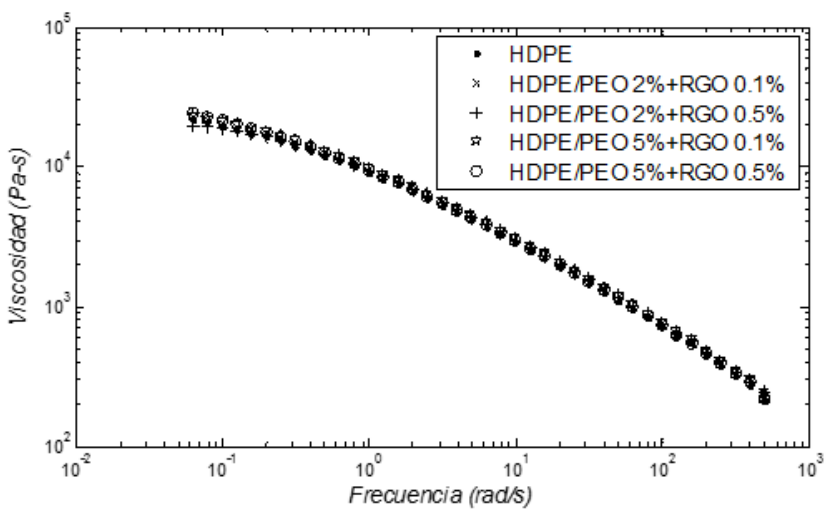

(b)

Fig. 6. Variación de la parte real de la viscosidad compleja con la frecuencia para los composites (a) Polipropileno/óxido de grafeno reducido-polióxido de etileno y (b) Polietileno de alta densidad/óxido de grafeno reducido-polióxido de etileno.

Se evaluó el efecto del rGO en el módulo de las poliolefinas, a temperaturas por debajo de su punto de fusión, usando el análisis dinámico mecánico en modo tensión desde una temperatura de 25 hasta $160^{\circ} \mathrm{C}$ para el PP y desde 25 hasta $120^{\circ} \mathrm{C}$ para el HDPE. La figura 7 muestra los resultados para los composites de PP con agente compatibilizante. En ella se aprecia claramente el efecto positivo del PEO, ya que, en los cuatro casos, el módulo elástico del composite es mucho mayor que el del polipropileno puro. Esto se hace más evidente a bajas temperaturas, donde el módulo tiene su valor máximo. A altas temperaturas, y a medida que la poliolefina se acerca a su punto de fusión, la diferencia entre los módulos tiende a desaparecer. La figura 8 muestra los valores del módulo a $30^{\circ} \mathrm{C}$, normalizándolo con el valor del mismo parámetro para el PP puro. Se puede apreciar que las muestras con $0.5 \%$ de rGO son las que tiene un valor mayor del módulo, 3.5 veces más que el PP puro para $2 \%$ de PEO y 2.9 veces más para la muestra con $5 \%$ de PEO.

Para el caso del HDPE, se aprecia un comportamiento muy similar al del PP, es decir, el módulo elástico del composite, con agente compatibilizante y a bajas temperaturas, es mayor que el del HDPE puro. En efecto, a una temperatura de $30^{\circ} \mathrm{C}$, el módulo de la muestra con 0.1 de rGO y $2 \%$ de PEO es 3.8 veces mayor que el del HDPE y con la misma cantidad de rGO, pero con 5\% de PEO el incremento es de 2.78 veces. Los composites con $0.5 \%$ de rGO presentaron un módulo muy similar al obtenido para la muestra PE15, como se muestra en la figura 10. Este comportamiento, tanto para el PP como para el HDPE, se puede explicar considerando que el PEO mejora la adhesión interfacial, lo cual es una manera muy eficiente de mejorar la interacción entre el rGO y la matriz poliolefínica. ${ }^{24}$ 
En los composites de PP y HDPE a los que no se les agregó PEO se puede apreciar que el módulo de la poliolefina pura es mayor que el de los composites con diferentes cantidades de rGO, lo cual es indicio de la nula interacción entre las matrices plásticas y el grafeno reducido, comportándose este último como impureza, ya que a mayor cantidad de rGO, menor el valor del módulo. Esta situación se revierte con el agente compatibilizante, tal y como queda evidenciado en las figuras 8 y 10.

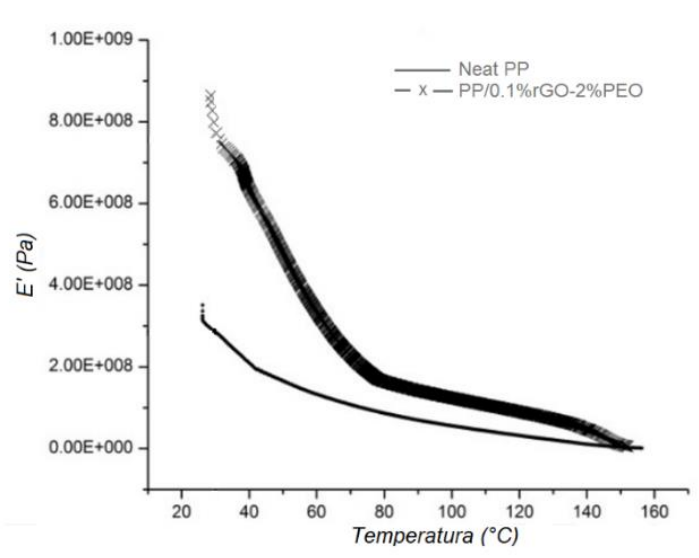

(a)

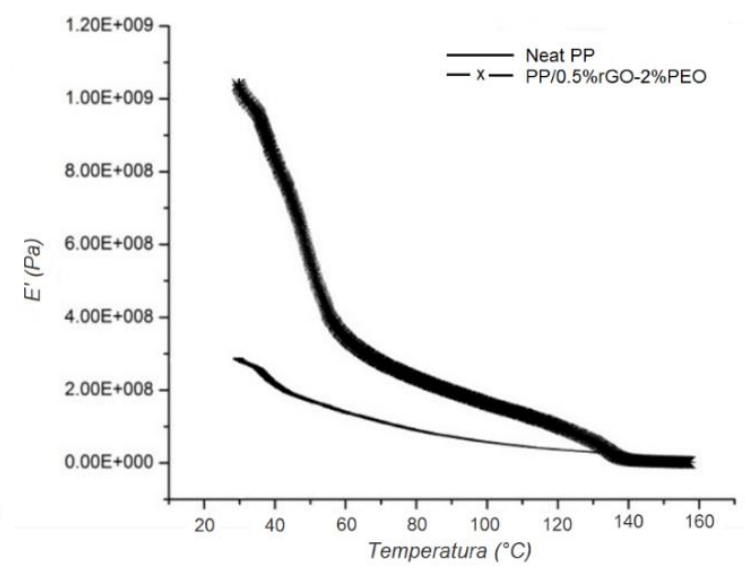

(b)

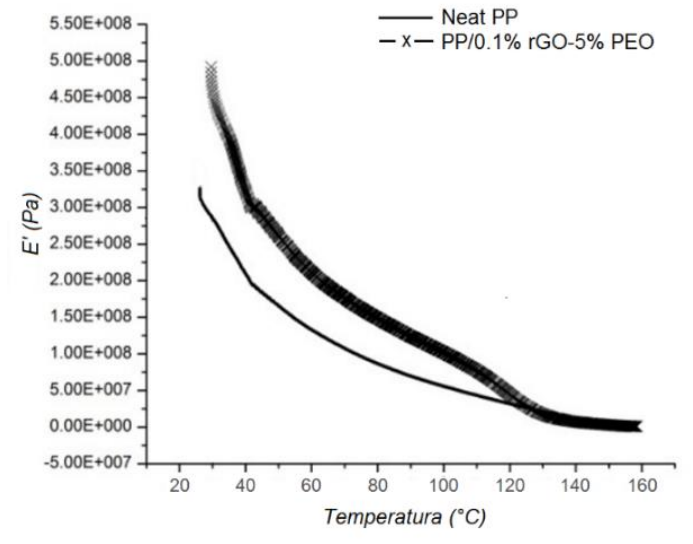

(c)

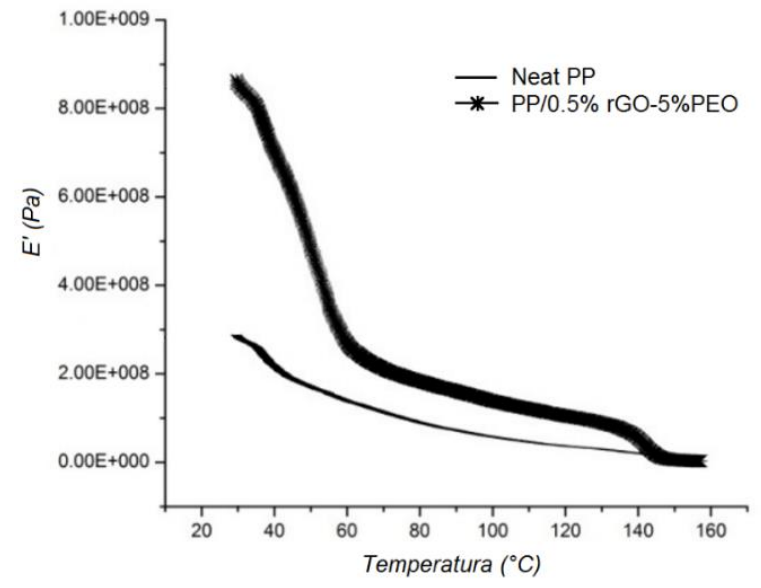

(d)

Fig. 7. Módulo elástico de los composites PP/rGO-PEO en función de la temperatura. 


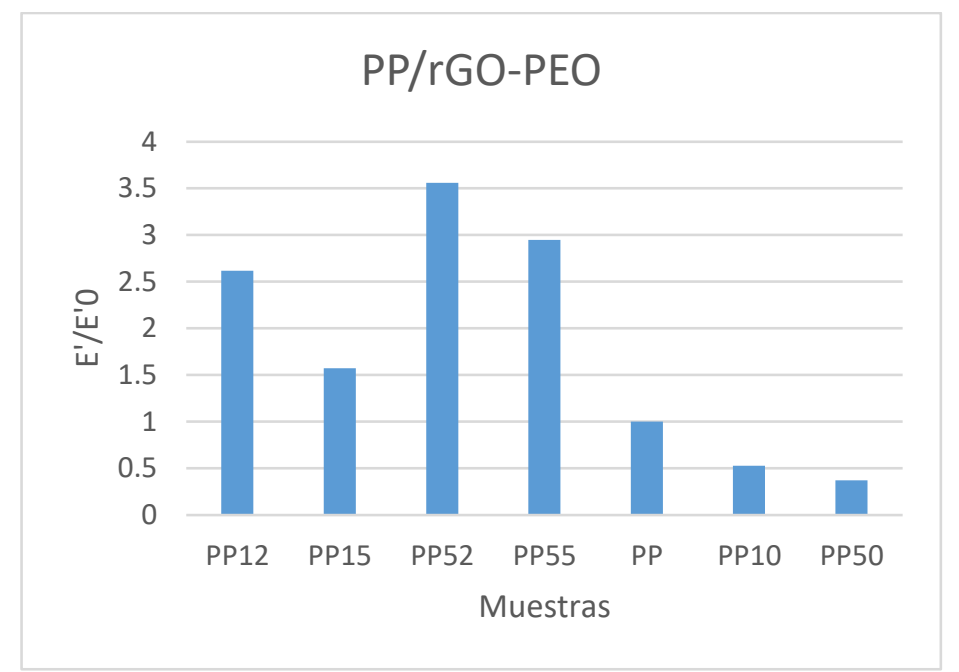

Fig. 8. Módulo elástico normalizado a $30^{\circ} \mathrm{C}$ para las muestras PP/rGO-PEO. E' ${ }_{0}$ corresponde al valor del módulo del PP puro.
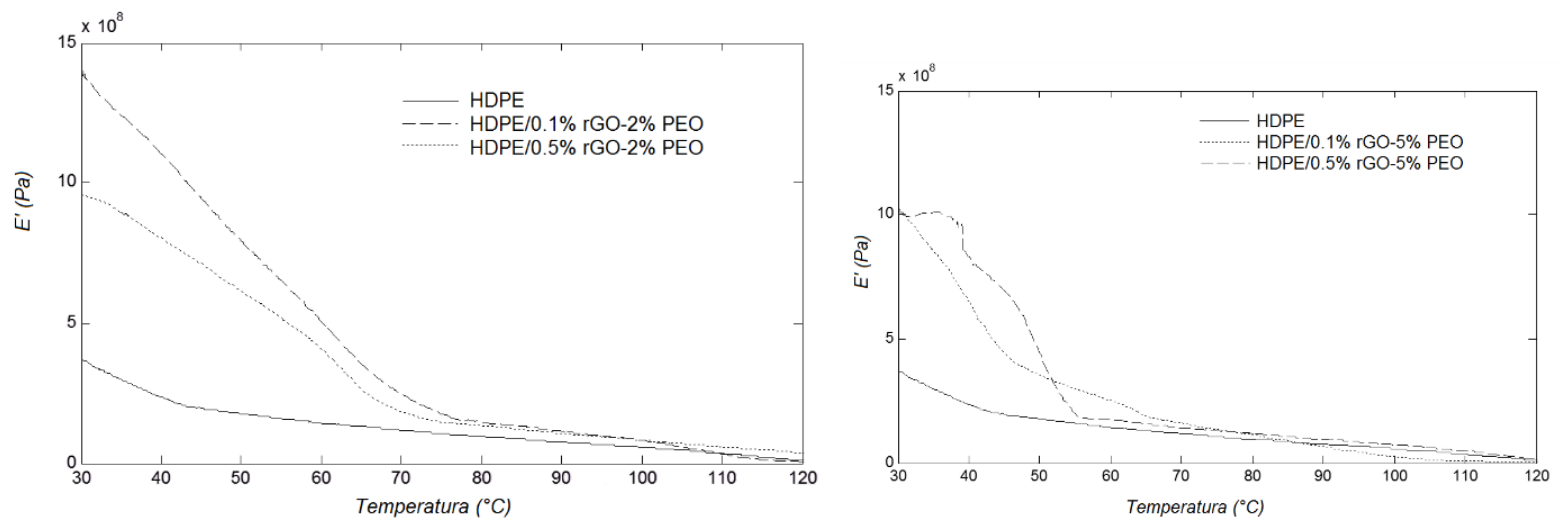

Fig. 9. Módulo elástico para los composites HDPE/rGO-PEO en función de la temperatura.

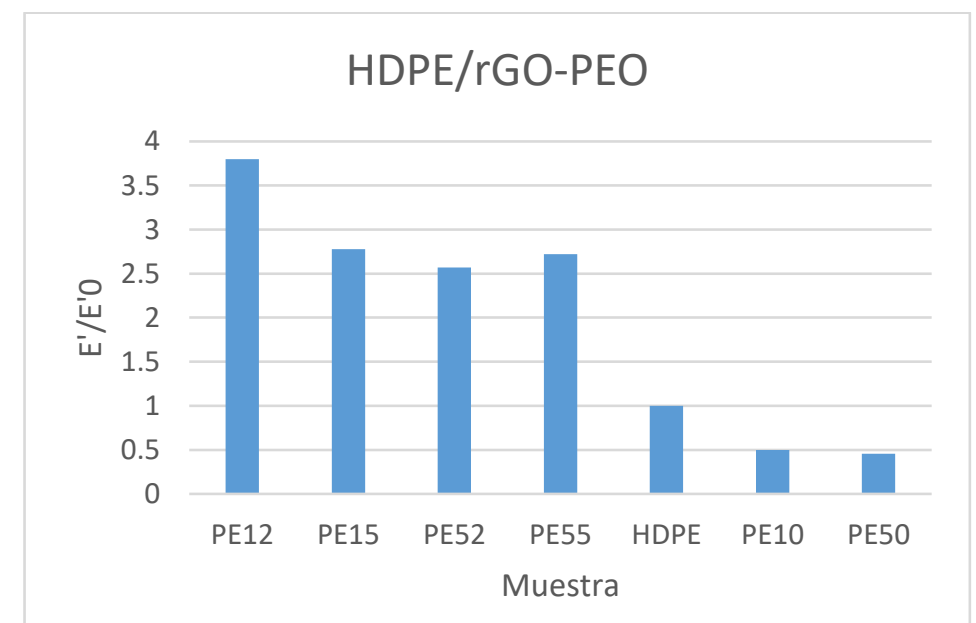

Fig. 10. Módulo elástico normalizado a $30^{\circ} \mathrm{C}$ para las muestras HDPE/rGO-PEO. E'o corresponde al valor del módulo del HDPE puro. 


\section{CONCLUSIONES}

Se prepararon composites de PP y HDPE con diferentes concentraciones de óxido de grafeno reducido con y sin un agente compatibilizante. Los resultados de la viscosidad dinámica no reflejan ningún efecto del rGO y/o del compatibilizante en la matriz poliolefínica, ya que las curvas obtenidas son muy similares en todos los casos. Probablemente con porcentajes mayores de rGO se podría apreciar mucho mejor su efecto en esta propiedad. Para el caso del módulo elástico, las cantidades añadidas del rGO no actúan como refuerzo, sino como impurezas, ya que el valor de la propiedad medida en los composites es menor a la de la poliolefina pura. Esta situación cambia al agregar el PEO, ya que en el composite con porcentajes de rGO tan bajos como $0.1 \%$ y $2 \%$ de PEO, se obtienen valores de casi cuatro veces el módulo del HDPE puro y de casi tres veces el módulo del PP puro. Al aumentar el porcentaje de rGO a $0.5 \%$ se obtienen valores del módulo alrededor de tres veces más altos para los composites que para las poliolefinas puras.

\section{BIBLIOGRAFÍA}

1. Cornelia Vasile, Handbook of Polyolefins. 2nd Ed., Revised and Expanded, Marcel Dekker, New York, USA (2000).

2. Andrew J. Peacock, Handbook of Polyethylene. Structures, Properties and Applications, Marcel Dekker, New York, USA (2000).

3. Clive Maier, Teresa Calafut, Polypropylene. The Definitive User's Guide and Databook, Plastic Design Library, New York, USA (1998).

4. Ehsan Bafekrpour (Ed.), Advanced Composite Materials: Properties and Applications, De Gruyter Open Ltd, Berlin, Germany (2017).

5. Deborah D. L. Chung, Composite Materials. Science and Applications. 2nd Ed., Springer Verlag, London (2010).

6. Klaus Friedrich, Stoyko Fakirov, Zhong Zhang, Polymer Composites. From Nano to Macro Scale, Springer, New York, USA (2005).

7. I. Krupa, I. Novák, I. Chodák, Electrically and thermally conductive polyethylene/graphite composites and their mechanical properties, Synthetic Metals 145 (2004) 245-252.

8. A Marcincin et al, Spinning, structure and properties of PP/CNTs and PP/carbon black composite fibers, 2014 IOP Conf. Ser.: Mater. Sci. Eng. 64012004.

9. Zdenko Spitalsky, Dimitrios Tasisb, Konstantinos Papagelis, Costas Galiotis, Carbon nanotube polymer composites: Chemistry, processing, mechanical and electrical properties, Progress in Polymer Science 35 (2010) 357-401

10. Maziyar Sabet, Hassan Soleimani, Mechanical and electrical properties of low density polyethylene filled with carbon nanotubes 2014 IOP Conf. Ser.: Mater. Sci. Eng. 64012001.

11. Saibom Park, Siyao He, Jianeng Wang, Andreas Stein, Christopher W. Macosko, Graphenepolyethylene nanocomposites: Effect of graphene functionalization, Polymer 104 (2016) 1-9.

12. Huanmin Li, Xu-Ming Xie, Polyolefin-functionalized graphene oxide and its GO/HDPE nanocomposite with excellent mechanical properties, Chinese Chemical Letters 29 (2018) 161-165.

13. Bin Wang, Dan Peng, Ruihua Lv, Bing Na, Hesheng Liu, Zhong Yu, Generic melt compounding strategy using reactive graphene towards high performance polyethylene/graphene nanocomposites, Composites Science and Technology 177 (2019) 1-9

14. Muhammad Z. Iqbal, Ahmed A. Abdala, Vikas Mittal, Sönke Seifert, Andrew M. Herring, Matthew W. Liberatore, Processable conductive graphene/polyethylene nanocomposites: 
Effects of graphene dispersion and polyethylene blending with oxidized polyethylene on rheology and microstructure, Polymer 98 (2016) 143-155.

15. Hongyu Chen, Valeriy V. Ginzburg, Jian Yang, Yunfeng Yang, Wei Liu,Yan Huang, Libo Du, Bin Chen, Thermal conductivity of polymer-based composites: Fundamentals and applications, Progress in Polymer Science 59 (2016) 41-85.

16. A. H. Castro Neto, F. Guinea, N. M. R. Peres, K. S. Novoselov, A. K. Geim, The electronic properties of graphene, Rev. Mod. Phys (2009) Vol. 81, No. 1 pp 109-162

17. Carlos A. Guerrero Salazar, Paloma B. Jiménez Vara, Virgilio A. González González, Tania E. Guerrero Salas, Composites de polietilen tereftalato (PET) con óxido de grafeno reducido (rGO), a ser publicado en Ingenierías (2019).

18. Mayra I. Llamas Hernández, Carlos A. Guerrero Salazar, Martín E. Reyes Melo, Juan F. Luna Martínez, Comportamiento elástico y morfológico de compuestos polipropileno-grafeno, Ingenierías (2015) Vol XVIII, 68, pp 32-40

19. Daniela C. Marcano, Dmitry V. Kosynkin, Jacob M. Berlin, Alexander Sinitskii, Zhengzong Sun, Alexander Slesarev, Lawrence B. Alemany, Wei Lu, James M. Tour, Improved Synthesis of Graphene Oxide, ACS Nano, Vol. 4, No. 8, 4806-4814, 2010.

20. Hua Bai, Chun Li, Gaoquan Shi, Functional Composite Materials Based on Chemically Converted Graphene, Adv. Mater. 2011, 23, 1089-1115.

21. Jianfeng Shen, Yizhe Hu, Min Shi, Xin Lu, Chen Qin, Chen Li, Mingxin Ye, Fast and Facile Preparation of Graphene Oxide and Reduced Graphene Oxide Nanoplatelets, Chem. Mater. 2009, 21, 3514-3520.

22. L. Stobinski, B. Lesiak, A. Malolepszy, M. Mazurkiewicz, B. Mierzwa, J. Zemek, P. Jiricek, I. Bieloshapka, Graphene oxide and reduced graphene oxide studied by the XRD, TEM and electron spectroscopy methods, Journal of Electron Spectroscopy and Related Phenomena 195 (2014) 145-154.

23. D.P. Hansora, N.G. Shimpi, S. Mishra, Graphite to Graphene via Graphene Oxide: An Overview on Synthesis, Properties, and Applications, JOM, Vol. 67, No. 12, 2015, pp 2855-2868.

24. Huanmin Li, Xu-Ming Xie, Polyolefin-functionalized graphene oxide and its GO/HDPE nanocomposite with excellent mechanical properties, Chinese Chemical Letters 29 (2018) 161165 . 